\title{
Continuum electrodynamics and the Abraham-Minkowski momentum controversy
}

\author{
Michael E. Crenshaw* \\ US Army Aviation and Missile Research, Development, and Engineering Center \\ RMDR-WDS-W, Redstone Arsenal, AL 35898, USA
}

[Published in Optical Trapping and Optical Micromanipulation XII, Proc. SPIE 9548, 95480J (25 Aug 2015)]

\begin{abstract}
Continuum electrodynamics is an axiomatic formal theory based on the macroscopic Maxwell equations and the constitutive relations. We apply the formal theory to a thermodynamically closed system consisting of an antireflection coated block of dielectric situated in free-space and illuminated by a quasimonochromatic field. We show that valid theorems of the formal theory are proven false by relativity and by conservation laws. Then the axioms of the formal theory are proven false at a fundamental level of mathematical logic. We derive a new formal theory of continuum electrodynamics for macroscopic electric and magnetic fields in a four-dimensional flat non-Minkowski material spacetime in which the speed of light is $c / n$.
\end{abstract}

Keywords: Classical Electrodynamics, Maxwell's equations, energy-momentum tensor, AbrahamMinkowski controversy

\section{MACROSCOPIC MAXWELL'S EQUATIONS AND RELATIVITY}

By the middle of the 19th century, the results of more than two centuries of electric and magnetic experiments had been codified into a set of equations bearing the names of Gauss, Ampère, Faraday, and, occasionally, Thompson. In 1864, Maxwell postulated a correction to the Ampère equation making it consistent with timedependent fields and leading to the prediction of electromagnetic wave phenomena. In honor of his achievement, the empirical equations of electrodynamics are known collectively as the Maxwell equations and are regarded as physical laws. Maxwell's equations appear in a microscopic form that applies to electric and magnetic fields in the vacuum and appear, separately, in a macroscopic form as the equations of motion for macroscopic electric and magnetic fields in continuous media. Then the macroscopic Maxwell equations, along with the constitutive relations, are the axioms of theoretical continuum electrodynamics. New theorems are derived by applying the ordinary operations of algebra, linear algebra, and calculus to the axioms and subsequent theorems. The axioms and the rules for combining axioms and theorems to form new theorems constitute an axiomatic formal theory of continuum electrodynamics.

The axiomatic formal theory of continuum electrodynamics is based on the macroscopic Maxwell (MaxwellHeaviside-Minkowski) equations

$$
\begin{gathered}
\nabla \times \mathbf{H}-\frac{1}{c} \frac{\partial \mathbf{D}}{\partial t}=0 \\
\nabla \cdot \mathbf{B}=0 \\
\nabla \times \mathbf{E}+\frac{1}{c} \frac{\partial \mathbf{B}}{\partial t}=0 \\
\nabla \cdot \mathbf{D}=0
\end{gathered}
$$

*michael.e.crenshaw4.civ@mail.mil 
and the linear constitutive relations

$$
\begin{aligned}
\mathbf{D} & =\varepsilon \mathbf{E} \\
\mathbf{B} & =\mu \mathbf{H} .
\end{aligned}
$$

We treat the case of a thermodynamically closed system consisting of a quasimonochromatic optical pulse incident on a simple linear dielectric. The otherwise homogeneous dielectric is draped with a gradient-index antireflection coating. The frequency of the incident radiation is required to be sufficiently far from resonance that absorption is negligible and dispersion is treated parametrically. Then the macroscopic refractive index, $n$, is a time-independent real scalar function of position. The constitutive relations become

$$
\begin{gathered}
\mathbf{D}=n^{2} \mathbf{E} \\
\mathbf{B}=\mathbf{H}
\end{gathered}
$$

by setting $\mu=1$ and $\varepsilon=(n(\mathbf{r}))^{2}$ in Eqs (1.2). These simple material properties can be regarded as additional axioms in our formal theory. As a matter of clarity and concision, we are only treating simple linear dielectrics. Then, we can treat Eqs. (1.3) as replacements for the axioms given by Eqs. (1.2). The constitutive relations, Eqs. (1.3), are used to substitutionally eliminate $\mathbf{H}$ and $\mathbf{D}$ from Eqs. (1.1). The macroscopic Maxwell equations are

$$
\begin{gathered}
\nabla \times \mathbf{B}-\frac{n^{2}}{c} \frac{\partial \mathbf{E}}{\partial t}=0 \\
\nabla \cdot \mathbf{B}=0 \\
\nabla \times \mathbf{E}+\frac{1}{c} \frac{\partial \mathbf{B}}{\partial t}=0 \\
\nabla \cdot\left(n^{2} \mathbf{E}\right)=0
\end{gathered}
$$

for a simple linear dielectric medium. After making the indicated substitutions, we commute the timeindependent refractive index with the temporal derivative in the Maxwell-Ampère law, Eq. (1.4a), and multiply the Faraday law, Eq. (1.4c), by $n$. Applying simple vector identities to commute curl and divergence operators with a scalar function of position, we obtain

$$
\begin{gathered}
\nabla \times \mathbf{B}-\frac{n}{c} \frac{\partial n \mathbf{E}}{\partial t}=0 \\
\nabla \cdot \mathbf{B}=0 \\
\nabla \times n \mathbf{E}+\frac{n}{c} \frac{\partial \mathbf{B}}{\partial t}=\frac{\nabla n}{n} \times n \mathbf{E} \\
\nabla \cdot n \mathbf{E}=-\frac{\nabla n}{n} \cdot n \mathbf{E} .
\end{gathered}
$$

Note that Eqs. (1.5) are valid theorems of the axiomatic formal theory of continuum electrodynamics. There are no assumptions or hidden axioms that enter the derivation of Eqs. (1.5) from the stated axioms, Eqs. (1.1) with the constitutive relations, Eqs. (1.3). 
There appears to be nothing remarkable about Eqs. (1.5) because they are clearly equivalent to Eqs. (1.1) with (1.3). On the other hand, it has been suggested [1] that Eqs. (1.5) violate the theory of special relativity. The Lorentz factor that is associated with Minkowski spacetime is

$$
\gamma_{M}=\frac{1}{\sqrt{1-\frac{v^{2}}{c^{2}}}} .
$$

This is true for fields in a dielectric medium [2], as well as for fields in the vacuum, and is validated by the Fizeau experiment [3]. Meanwhile, the material Lorentz factor [4, 5],

$$
\gamma_{d}=\frac{1}{\sqrt{1-\frac{n^{2} v^{2}}{c^{2}}}}
$$

is associated with Eqs. (1.5) and can be considered to be incorrect based on the correct Lorentz factor, Eq. (1.6). Now, the two sets of equations, Eqs. (1.5) and Eqs. (1.1) with (1.3), are equivalent within the current theory of continuum electrodynamics and are equally correct. Because the two sets of equations have different relativistic properties, at least one set of equations is wrong. Then both sets of equations are wrong by equivalence. Consequently, both the valid theorem of continuum electrodynamics, Eqs. (1.5), and the axioms of continuum electrodynamics, Eqs. (1.1) and (1.3), are false. This is a mathematical fact.

\section{THE ABRAHAM-MINKOWSKI CONTROVERSY}

In 1908, Minkowski [6] proposed an energy-momentum tensor for electromagnetic fields in a linear medium. The following year, Abraham [7] argued from symmetry to propose a correction to the momentum density that appears in the Minkowski energy-momentum tensor. The "corrected" tensor is known as the Abraham energy-momentum tensor. The consequent Abraham-Minkowski controversy refers to the century-long inability of scientists to conclusively identify the role of electromagnetic momentum for the propagation of light in linear media 8 12]. To be sure, the total momentum [12 14],

$$
\mathbf{G}_{T}=\int_{\sigma} \frac{n \mathbf{E} \times \mathbf{B}}{c} d v,
$$

for a quasimonochromatic pulse incident on a rare or antireflection-coated transparent dielectric has never been in doubt because the momentum density integrated over a region that is extended to all-space $\sigma$ is conserved in a closed system. Instead, the disputed issue is the amount of the total momentum that remains with the propagating electromagnetic field and the amount of total momentum that is transferred to the matter by various forms of radiation pressure.

The Abraham-Minkowski momentum controversy is very much a part of axiomatic continuum electrodynamics. We want to revisit the electromagnetic continuity equations from the point of view of the axiomatic formal theory presented in Section I. Subtracting the scalar product of Eq. (1.4a) with E from the scalar product of Eq. (1.4c) with B, one obtains

$$
\frac{1}{c} \frac{\partial}{\partial t}\left[\frac{1}{2}\left(n^{2} \mathbf{E}^{2}+\mathbf{B}^{2}\right)\right]+\nabla \cdot c \frac{\mathbf{E} \times \mathbf{B}}{c}=0 .
$$

Known as Poynting's theorem, Eq. (2.2) has the appearance of being an energy continuity equation. Similarly, we can start with the vector product of $n^{2} \mathbf{E}$ with Eq. (1.4c) and subtract the vector product of Eq. (1.4a) with B to obtain

$$
\frac{1}{c} \frac{\partial\left(n^{2} \mathbf{E} \times \mathbf{B}\right)}{\partial t}+\nabla \cdot \mathbf{W}=\mathbf{f}_{M}
$$

which has the appearance of a momentum continuity equation. Here,

$$
W_{i j}=-n^{2} E_{i} E_{j}-B_{i} B_{j}+\frac{1}{2}\left(n^{2} \mathbf{E}^{2}+\mathbf{B}^{2}\right) \delta_{i j}
$$


is the Maxwell stress tensor and

$$
\mathbf{f}_{M}=-\frac{n^{2} \mathbf{E}^{2}}{2} \frac{\nabla\left(n^{2}\right)}{n^{2}}
$$

is a force density. Both Eq. (2.2) and Eq. (2.3) are valid theorems of continuum electrodynamics. As a matter of linear algebra, the scalar equation, Eq. (2.2), and the three orthogonal components of the vector equation, Eq. (2.3), can be written as a single matrix differential equation

$$
\partial_{\beta} T_{M}^{\alpha \beta}=f_{M}^{\alpha} .
$$

In Eq. (2.6),

$$
\partial_{\beta}=\left(\frac{1}{c} \frac{\partial}{\partial t}, \frac{\partial}{\partial x}, \frac{\partial}{\partial y}, \frac{\partial}{\partial z}\right)
$$

is an operator,

$$
T_{M}^{\alpha \beta}=\left[\begin{array}{cccc}
\left(n^{2} \mathbf{E}^{2}+\mathbf{B}^{2}\right) / 2 & (\mathbf{E} \times \mathbf{B})_{1} & (\mathbf{E} \times \mathbf{B})_{2} & (\mathbf{E} \times \mathbf{B})_{3} \\
\left(n^{2} \mathbf{E} \times \mathbf{B}\right)_{1} & W_{11} & W_{12} & W_{13} \\
\left(n^{2} \mathbf{E} \times \mathbf{B}\right)_{2} & W_{21} & W_{22} & W_{23} \\
\left(n^{2} \mathbf{E} \times \mathbf{B}\right)_{3} & W_{31} & W_{32} & W_{33}
\end{array}\right]
$$

is a matrix, and

$$
f_{M}^{\beta}=\left(0,-\frac{(n \mathbf{E})^{2}}{2} \frac{\nabla\left(n^{2}\right)}{n^{2}}\right)^{T}
$$

is a matrix.

The matrix, Eq. (2.8), which has the appearance of being an energy-momentum tensor is known as the Minkowski energy-momentum tensor. In a thermodynamically closed system, the energy-momentum tensor is required to be diagonally symmetric so that angular momentum is conserved [15, 16]. The Minkowski energymomentum tensor is not symmetric and Abraham proposed a diagonally symmetric matrix

$$
T_{A}^{\alpha \beta}=\left[\begin{array}{cccc}
\left(n^{2} \mathbf{E}^{2}+\mathbf{B}^{2}\right) / 2 & (\mathbf{E} \times \mathbf{B})_{1} & (\mathbf{E} \times \mathbf{B})_{2} & (\mathbf{E} \times \mathbf{B})_{3} \\
(\mathbf{E} \times \mathbf{B})_{1} & W_{11} & W_{12} & W_{13} \\
(\mathbf{E} \times \mathbf{B})_{2} & W_{21} & W_{22} & W_{23} \\
(\mathbf{E} \times \mathbf{B})_{3} & W_{31} & W_{32} & W_{33}
\end{array}\right]
$$

for the energy-momentum tensor. Although physically motivated by conservation laws, Abraham's conjecture creates other conservation issues with the formal theory of continuum electrodynamics.

The modern consensus is that the system represented by the macroscopic Maxwell theory is not thermodynamically closed, but is, in fact, an electromagnetic subsystem that is coupled to a material subsystem. In this scenario, the Minkowski force density $f_{M}^{\alpha}$ transfers momentum from the electromagnetic subsystem, $T$, to a material subsystem, $R$. Then,

$$
\partial_{\beta} R^{\alpha \beta}=-f_{M}^{\alpha}
$$

such that the total field plus material system thermodynamically closed,

$$
\partial_{\beta}\left(T_{M}^{\alpha \beta}+R^{\alpha \beta}\right)=0
$$

Then, motivated by the conservation laws, one assumes some properties for the material subsystem $R$. In Pfeifer, et al. [12], for example,

$$
R^{\alpha \beta}=\left[\begin{array}{cccc}
c^{2} \rho & c \rho v_{1} & c \rho v_{2} & c \rho v_{3} \\
c \rho v_{1}+\left(1-n^{2}\right)(\mathbf{E} \times \mathbf{B})_{1} & \rho v_{1} v_{1} & \rho v_{1} v_{2} & \rho v_{1} v_{3} \\
c \rho v_{2}+\left(1-n^{2}\right)(\mathbf{E} \times \mathbf{B})_{2} & \rho v_{2} v_{1} & \rho v_{2} v_{2} & \rho v_{2} v_{3} \\
c \rho v_{3}+\left(1-n^{2}\right)(\mathbf{E} \times \mathbf{B})_{3} & \rho v_{3} v_{1} & \rho v_{3} v_{2} & \rho v_{3} v_{3}
\end{array}\right]
$$


where $\rho$ is the mass density and $\mathbf{v}$ is the velocity field. In order for the total momentum, Eq. (2.1), to be conserved, we must assume the material momentum density to be 12 ]

$$
\rho \mathbf{v}=(n-1) \frac{\mathbf{E} \times \mathbf{B}}{c} .
$$

Now, $f_{M}^{\alpha}$ can be made vanishingly small for an arbitrarily large homogeneous medium draped with a gradientindex antireflection coating. In that limit, the electromagnetic and material subsystems are not coupled and, consequently,

$$
\begin{gathered}
\partial_{\beta} T_{M}^{\alpha \beta}=0 \\
\partial_{\beta} R^{\alpha \beta}=0 .
\end{gathered}
$$

Then the material tensor

$$
R^{\alpha \beta}=\left[\begin{array}{cccc}
c^{2} \rho & c \rho v_{1} & c \rho v_{2} & c \rho v_{3} \\
c \rho v_{1} & \rho v_{1} v_{1} & \rho v_{1} v_{2} & \rho v_{1} v_{3} \\
c \rho v_{2} & \rho v_{2} v_{1} & \rho v_{2} v_{2} & \rho v_{2} v_{3} \\
c \rho v_{3} & \rho v_{3} v_{1} & \rho v_{3} v_{2} & \rho v_{3} v_{3}
\end{array}\right]
$$

is the dust tensor that is the total energy-momentum tensor for the unimpeded (force-free) flow of noninteracting material particles. Furthermore, the vanishingly small force density means that there is no appreciable source or sink of momentum. Consequently, the Minkowski tensor is the total energy-momentum tensor of a thermodynamically closed system and that means that the Minkowski momentum

$$
\mathbf{G}_{M}=\int_{\sigma} \frac{n^{2} \mathbf{E} \times \mathbf{B}}{c} d v
$$

is conserved. Although the mathematics, $\partial_{\beta} T_{M}^{i \beta}=0$, tell us that the Minkowski momentum is conserved, it is well-known that the Minkowski momentum is a factor of $n$ greater than the incident momentum [12 14]. Then the continuity equation, Eq. (2.6), is false in the limit of an arbitrarily large homogenous medium draped with a gradient-index antireflection coating. Because a valid theorem of continuum electrodynamics, Eqs. (2.6), has been proven false, the axioms of the theory, the macroscopic Maxwell equations, are also false. In addition, the absence of diagonal symmetery and the consequent lack of angular momentum conservation also disproves the macroscopic Maxwell equations by disproving a valid theorem that has been derived from these axioms.

The entire history of the Abraham-Minkowski controversy is the search for valid conservation laws for energy and momentum. Recasting the debate in the language of axioms and formal logic imposes the discipline that is necessary in order to prevent the introduction of hypothetical forces and momentums. Instead, the Maxwellian theory is formally proven to be invalid because it leads to demonstrably incorrect results. The search for valid conservation laws for energy and momentum must be taken outside the Maxwellian, and equivalent, formulations of continuum electrodynamics.

\section{WHAT TO DO?}

The phenomenological Maxwell-Heaviside-Minkowski equations have carried us through a century-and-ahalf of remarkable progress in science. However, we have demonstrated with examples from relativity and energy/momentum conservation that there are situations in which the axiomatic formal theory of continuum electrodynamics is contradicted. While we can, and do, work around these problems, we would seek a theory of continuum electrodynamics with innate conformity to other fundamental physical principles. Despite almost certain objections, there is now clear and compelling evidence of the need to a construct a completely new theory of continuum electrodynamics. Ideally, the best place to start is with one of the other fundamental physical principles, like conservation, relativity, or field theory. While these principles are intrinsic to the vacuum, the implementations of these fundamental principles in a dielectric environment lead to dogmatic arguments about 
the momentum of electromagnetic fields and a perpetuation of the Abraham-Minkowski momentum controversy. Then we must jettison the phenomenological Maxwell equations, the constitutive relations, and the variations of the macroscopic Maxwell equations. It is necessary to derive the equations of motion for macroscopic fields and to re-derive the other fundamental physical principles for fields in a region of space in which the speed of light is $c / n$.

We define an inertial reference frame $S(x, y, z)$ that is located in free-space. If a light pulse is emitted from the origin at time $t=0$, then wavefronts are described by a mathematical formula

$$
x^{2}+y^{2}+y^{2}-(c t)^{2}=0 .
$$

The four-vector $\left(x_{0}, x, y, z\right)$ represents the position of a point in a four-dimensional Minkowski vacuum spacetime in which $x_{0}=c t$ is a timelike coordinate. Dielectrics are mostly vacuum, consisting of tiny bits of matter separated by relatively large distances. Very small particles, like electrons, can travel through the dielectric at speeds greater than $c / n$ because they mostly pass through the interstitial space. Likewise, the instantaneous speed of light in a dielectric is $c$, although the effective speed of light is $c / n$ due to scattering. In continuum electrodynamics, however, a dielectric is continuous at all length scales. In the rest frame of the dielectric, light travels at a uniform speed of $c / n$ and wavefronts from a point light source are described by

$$
x^{2}+y^{2}+y^{2}-\left(\frac{c t}{n}\right)^{2}=0 .
$$

The four-vector $\left(\bar{x}_{0}, x, y, z\right)$ represents the position of a point in a flat four-dimensional non-Minkowski material spacetime in which $\bar{x}_{0}=c t / n$ is a timelike coordinate. Although the existence of a non-Minkowski material spacetime might be viewed skeptically, Eq. (3.2), which defines the spacetime, is solid ground.

If we adopt Minkowski spacetime, the Lagrange equation for fields in a linear medium is [15, 17]

$$
\frac{d}{d x_{0}} \frac{\partial \mathcal{L}}{\partial\left(\partial A_{j} / \partial x_{0}\right)}=\frac{\partial \mathcal{L}}{\partial A_{j}}-\sum_{i} \partial_{i} \frac{\partial \mathcal{L}}{\partial\left(\partial_{i} A_{j}\right)} .
$$

Applying Eq. (3.3) to the Lagrangian density of the electromagnetic field in the medium results in the usual macroscopic Maxwell equations [15].

As discussed above, a dielectric environment is distinct from a vacuum environment. Adopting the nonMinkowski material spacetime, we derive [18]

$$
\frac{d}{d \bar{x}_{0}} \frac{\partial \mathcal{L}}{\partial\left(\partial A_{j} / \partial \bar{x}_{0}\right)}=\frac{\partial \mathcal{L}}{\partial A_{j}}-\sum_{i} \partial_{i} \frac{\partial \mathcal{L}}{\partial\left(\partial_{i} A_{j}\right)}
$$

as the Lagrange equation for electromagnetic fields in a linear medium. Applying Eq. (3.4) to the Lagrangian density of the electromagnetic field in the medium

$$
\mathcal{L}=\frac{1}{2}\left(\left(\frac{\partial \mathbf{A}}{\partial \bar{x}_{0}}\right)^{2}-(\nabla \times \mathbf{A})^{2}\right),
$$

one defines the canonical momentum density $\Pi$ in terms of components as

$$
\Pi_{j}=\frac{\partial \mathcal{L}}{\partial\left(\partial A_{j} / \partial \bar{x}_{0}\right)}=\frac{\partial A_{j}}{\partial \bar{x}_{0}}
$$

such that

$$
\boldsymbol{\Pi}=\frac{\partial \mathbf{A}}{\partial \bar{x}_{0}} .
$$

Then the Lagrange equation takes the familiar form of the wave equation

$$
\nabla \times(\nabla \times \mathbf{A})+\frac{\partial^{2} \mathbf{A}}{\partial \bar{x}_{0}^{2}}=0 .
$$


We define a macroscopic magnetic field

$$
\mathbf{B}=\nabla \times \mathbf{A}
$$

Substituting the macroscopic fields $\mathbf{B}$ and $\boldsymbol{\Pi}$ into the wave equation, Eq. (3.8), and performing vector calculus operations on the macroscopic fields, Eqs. (3.7) and (3.9), and the wave equation, we obtain

$$
\begin{gathered}
\nabla \times \mathbf{B}+\frac{\partial \mathbf{\Pi}}{\partial \bar{x}_{0}}=0 \\
\nabla \cdot \mathbf{B}=0 \\
\nabla \times \mathbf{\Pi}-\frac{\partial \mathbf{B}}{\partial \bar{x}_{0}}=\frac{\nabla n}{n} \times \mathbf{\Pi} \\
\nabla \cdot \boldsymbol{\Pi}=\frac{\nabla n}{n} \cdot \boldsymbol{\Pi} .
\end{gathered}
$$

as equations of motion for the macroscopic fields. It might appear that these equations are equal to the phenomenological Maxwell equations under a simple re-parameterization. However, this is where we need to be careful not to be dragged back into the discredited theory. Specifically, classical continuum electrodynamics admits improper tensor transformations of coordinates that alter the relativistic and conservation properties of the macroscopic Maxwell equations. We have abandoned classical continuum electrodynamic theory for that reason and any transformations of Eqs. (3.10) are required to maintain the tensor properties. In particular, the timelike coordinate $\bar{x}_{0}=c t / n$ must remain intact in order to preserve the spacetime. The derivation from field theory gives Eqs. (3.10) their privileged status and gives them physical relevance as equations of motion for macroscopic electromagnetic fields in a flat non-Minkowski material spacetime. The suggested reparameterization is forbidden.

\section{RESOLUTION OF THE ABRAHAM-MINKOWSKI CONTROVERSY}

The equations of motion for macroscopic fields in a continuous dielectric medium, Eqs. (3.10), are theorems of the field theory in a non-Minkowski material spacetime in which light travels at speed $c / n$. Now that the field theory has done its job and we have these theorems, we can designate them as the axioms of our new theory of continuum electrodynamics, keeping the field theory in the background. The equations of motion for the macroscopic fields, Eqs. (3.10), can be combined in the usual manner, using algebra and calculus, to write electromagnetic energy and momentum continuity equations

$$
\begin{gathered}
\frac{\partial}{\partial \bar{x}_{0}}\left[\frac{1}{2}\left(\boldsymbol{\Pi}^{2}+\mathbf{B}^{2}\right)\right]+\nabla \cdot(\mathbf{B} \times \boldsymbol{\Pi})=\frac{\nabla n}{n} \cdot(\mathbf{B} \times \mathbf{\Pi}) \\
\frac{\partial(\mathbf{B} \times \mathbf{\Pi})}{\partial \bar{x}_{0}}+\nabla \cdot \mathbf{W}=-\boldsymbol{\Pi}\left(\boldsymbol{\Pi} \cdot \frac{\nabla n}{n}\right),
\end{gathered}
$$

where

$$
W_{i j}=-\Pi_{i} \Pi_{j}-B_{i} B_{j}+\frac{1}{2}\left(\mathbf{\Pi}^{2}+\mathbf{B}^{2}\right) \delta_{i j}
$$

is the Maxwell stress-tensor. Then the continuity equations, Eqs. (4.1) and (4.2), can be written as

$$
\bar{\partial}_{\alpha} T^{\alpha \beta}=f^{\alpha}
$$


where

$$
T^{\alpha \beta}=\left[\begin{array}{cccc}
\left(\mathbf{\Pi}^{2}+\mathbf{B}^{2}\right) / 2 & (\mathbf{B} \times \mathbf{\Pi})_{1} & (\mathbf{B} \times \mathbf{\Pi})_{2} & (\mathbf{B} \times \mathbf{\Pi})_{3} \\
(\mathbf{B} \times \mathbf{\Pi})_{1} & W_{11} & W_{12} & W_{13} \\
(\mathbf{B} \times \mathbf{\Pi})_{2} & W_{21} & W_{22} & W_{23} \\
(\mathbf{B} \times \mathbf{\Pi})_{3} & W_{31} & W_{32} & W_{33}
\end{array}\right]
$$

is the energy-momentum tensor,

$$
\bar{\partial}_{\beta}=\left(\frac{\partial}{\partial \bar{x}_{0}}, \frac{\partial}{\partial x}, \frac{\partial}{\partial y}, \frac{\partial}{\partial z}\right)
$$

is the material four-divergence operator [5], and

$$
f^{\alpha}=\left(\frac{\nabla n}{n} \cdot(\mathbf{B} \times \mathbf{\Pi}),-\boldsymbol{\Pi}\left(\boldsymbol{\Pi} \cdot \frac{\nabla n}{n}\right)\right)
$$

is the four-force density.

The continuity equations, Eqs. (4.1), (4.2), and (4.4), are valid theorems of a continuum electrodynamics whose axioms, Eqs. (3.10), are themselves theorems of field theory in a flat non-Minkowski material spacetime. We can identify two limiting cases of particular interest. In the limit of piecewise-homogeneous media, the field passes from one simple linear dielectric (or the vacuum) into a second such medium through a planar interface at normal incidence where the field is connected by boundary conditions at the interfaces between homogeneous linear materials. There is radiation pressure on the surface due to Fresnel reflection. Here we consider the case of an arbitrarily large homogeneous simple linear dielectric draped with a gradient index antireflection coating. The gradient of the index of refraction $\nabla n$ can be made sufficiently small that reflections can be neglected [14]. It is in this limit of an unimpeded continuous flow of electromagnetic radiation in which there are no sources or sinks of energy or momentum that the energy and momentum densities can be expressed through continuity equations and a total energy-momentum tensor for a thermodynamically closed system. (Lorentz dipole forces belong to a microscopic model of non-interacting particles, not to the macroscopic dielectric considered here and certainly not to the axiomatic formalism of continuum electrodynamics.) Taking the force density to be negligible, we obtain an energy continuity equation and a momentum continuity equation

$$
\begin{gathered}
\frac{\partial}{\partial \bar{x}_{0}}\left[\frac{1}{2}\left(\boldsymbol{\Pi}^{2}+\mathbf{B}^{2}\right)\right]+\nabla \cdot(\mathbf{B} \times \mathbf{\Pi})=0 \\
\frac{\partial(\mathbf{B} \times \mathbf{\Pi})}{\partial \bar{x}_{0}}+\nabla \cdot \mathbf{W}=0
\end{gathered}
$$

expressing conservation of the total energy

$$
U=\int_{\sigma} \frac{1}{2}\left(\boldsymbol{\Pi}^{2}+\mathbf{B}^{2}\right) d v
$$

and the total momentum

$$
\mathbf{G}=\int_{\sigma} \frac{\mathbf{B} \times \mathbf{\Pi}}{c} d v
$$

in an unimpeded (force-free) continuous flow of light in a non-Minkowski material spacetime. It is easy to verify that these conserved quantities are indeed invariant in time, as is required. Likewise, in the limit that the surface four-force density can be neglected there is no coupling of energy or momentum to any material subsystem. Then, $T^{\alpha \beta}$, as shown in Eq. (4.5), is the total energy-momentum tensor of a thermodynamically closed system. As noted by Pfeifer, et al., [12] arbitrary partitions of the total energy-momentum tensor are not particularly useful because only the total energy-momentum tensor is physically relevant. 


\section{CONCLUDING SUMMARY}

Maxwellian continuum electrodynamics allows algebraic transformations that appear to be innocuous but change the conservation properties, the relativistic properties, and the spacetime embedding of the coupled equations of motion. Identifying an arbitrarily large region of space in which the velocity of light is $c / n$, instead of $c$, we defined a non-Minkowski material spacetime $\left(\bar{x}_{0}=c t / n, x, y, z\right)$. Applying field theory in the material spacetime, we derived equations of motion for the macroscopic electric and magnetic fields. Requiring that we maintain a time-like coordinate $\bar{x}_{0}=c t / n$ prevents improper transformations of coordinates.

[1] Private communications.

[2] Møller, C., The Theory of Relativity, 2nd ed., Oxford, 1952.

[3] Fizeau, M. H., "Sur les hypothèses relatives à l'èther lumineux," C. R. Acad. Sci. (Paris) 33, 349-355 (1851) (English Translation: Phil. Mag. 2, 568-573 (1851)).

[4] Rosen, N., "Special theories of relativity," Am. J. Phys 20, 161-164 (1952).

[5] Ravndal, F., "Effective electromagnetic theory for dielectric media," http://arxiv.org/pdf/0804.4013v3.pdf (2008).

[6] Minkowski, "Die Grundgleichungen für die elektromagnetischen Vorgänge in bewegten Körpern," H., Natches. Ges. Wiss. Göttingen 53-111 (1908).

[7] Abraham, M., "Zur Elektrodynamik bewegter Körper," Rend. Circ. Mat. Palermo 28, 1-28 (1909).

[8] Barnett, S. M., Loudon, R., "The enigma of optical momentum in a medium," Phil. Trans. A 368, 927-939 (2010).

[9] Mansuripur, M., Zakharian, A. R., "Maxwell's macroscopic equations, the energy-momentum postulates, and the Lorentz law of force," Phys. Rev. E 79, 026608 (2009).

[10] Milonni, P. W., Boyd, R. W., "Momentum of light in a dielectric medium," Adv. Opt. Photonics 2, 519-533 (2010).

[11] Kemp, B. A., "Resolution of the Abraham-Minkowski debate: Implications for the electromagnetic wave theory of light in matter," J. Appl. Phys. 109, 111101 (2011).

[12] Pfeifer, R. N. C., Nieminen, T. A., Heckenberg, N. R., Rubinsztein-Dunlop, H., "Colloquium: momentum of an electromagnetic wave in dielectric media," Rev. Mod. Phys. 79, 1197-1216 (2007).

[13] Gordon, J. P., "Radiation forces and momenta in dielectric media," Phys. Rev. A 8, 14-21 (1973).

[14] Crenshaw, M. E., Bahder, T. B., "Electromagnetic energy, momentum, and angular momentum in an inhomogeneous linear dielectric," Opt. Commun. 285, 5180-5183 (2012).

[15] Cohen-Tannoudji, C., Dupont-Roc, J., Grynberg, G., Photons and Atoms, Wiley (1989).

[16] Jackson, J. D., Classical Electrodynamics, 2nd ed., Wiley, 1975.

[17] Hillery, M., Mlodinow, L., "Quantization of electrodynamics in nonlinear dielectric media," Phys. Rev. A 55, 678-689 (1984).

[18] Crenshaw, M. E., "The Theory of Electrodynamics in a Linear Dielectric," http://arxiv.org/pdf/1303.1412v3.pdf (2014). 